\title{
Revista

\section{Cultura, mídia, sociedade: hipóteses sobre a construção social dos problemas sociais}

\section{Culture, media and society: hypothesis on the construction of social problems}

PIERFRANCO MALIZIA

Professor do Programa de Pós-Graduação em Comunicação da Libera Universitá Maria Ss. Assunta - LUMSA de Roma. <pfmalizia@yahoo.it>

\section{RESUMO}

Na reflexão sociológica, a partir dos anos 70, os problemas sociais suscitaram um interesse crescente solicitando, por consequência, um amplo debate, e foram inicialmente concebidos e tratados como "fatos existentes em si próprios" e só uma relativa reflexão acerca do que os levava exatamente a serem não só "problemáticos" (valorização social) e acerca dos métodos de construção social dos próprios. O artigo é uma tentativa de construir uma hipótese de trabalho relativa a como os prolemas sociais se tornam tais efetivamente ou talvez não muito, partindo como pressuposto da cultura,que transforma (ou não) eventos e coisas em objetos culturais com específicos significados no âmbito de cada cultura, e isso explica como alguns fenômenos do mundo social são considerados importantes, transformados em objetos culturais e finalmente em problemas sociais, enquanto outros permanecem no esquecimento.

Palavras-chave: Problemas sociais; Cultura; Sociedade; Mídia.

\begin{abstract}
In the sociological reflection, since the 1970's, social problems have raised interest, consequentially creating a deep debate, they were, first of all, conceived and studied as factors existing in itself; only a specific reflection will socially validate them, as facts not only problematic and with their own methods of social construction. This paper tries to build a work hypothesis on the real origin of social problems, assuming culture as the founding element, that transforms (or not) events and things in cultural objects with a specific significance in the field of each culture; this explains how some phenomena of the social world are considered important, becoming cultural objects and finally social problems, while others completely fall in the oblivion.
\end{abstract}

KEywords: Social problems; Culture; Society; Media. 


\section{Os problemas sociais: uma introdução}

— timologicamente falando, problema indica uma dificuldade, um obstáculo e, nas

¿iências filosóficas, o termo designa uma espécie de paragem do pensamento perante algo que impede, naquele momento, o prosseguimento do mesmo pensamento conforme a perspectiva inicia. Portanto, com a atribuição do complemento social, podemos definir, em primeira abordagem, os problemas sociais como impedimento à permanência do normal andamento da vida social.

Na sociologia da cultura, a partir dos anos 1970, os problemas sociais suscitaram um interesse crescente. Solicitando, por consequência, um amplo debate, e foram inicialmente concebidos e tratados como fatos existentes em si próprios e só uma relativa reflexão acerca do que os levava exatamente a serem não só problemáticos (valorização social) e acerca dos métodos de construção social dos próprios problemas (produto cultural).

Por outras palavras, é a partir dos anos 70 (em diante), no âmbito mais geral do chamado social costructionism (Pawlunch, 1996), que os problemas socias são analizados mais em termos (Sills, 1968) de processo do que de condição e, por consequência, com uma progressiva mudança do discurso acerca de como e porque a sociedade em primeiro lugar define uma situação ou, melhor, estigmatiza-a (Goffman, 1970) e, finalmente vive-a (e deixa-as viver, referindo-nos às arenas) em termos de significação e de vivência sociocultural; ainda Goffman acrescenta que a possibilidade real de existência das situações será diretamente proporcional ao "poder das pessoas que elaboram a definição de impor a sua própria definição aos outros; a estigmatização é um processo que um grupo de pessoas impõe a outro grupo" (Berger-Berger, 1995, p. 348). Tendo isso em conta, devemos, porém, lembrar que este poder (que Lewis Carrol em Alice no País das Maravilhas já reconhecia como forte) é efetivo só na medida em que a sociedade globalmente com os seus processos de construção social da realidade 
objetivam a mesma, fixando-a de maneira aparentemente imanente: "os homens em conjunto produzem um ambiente humano" (Berger-Luckmann, 1969, p. 79). E bastaria apenas essa citação para aludir imediatamente ao fato de como esta realidade possa ser bastante precária, consideração que, devido aos problemas sociais (em termos quer de definição, quer de consequências), reveste uma importância absoluta. Parafraseando Becker (1987, p. 9) poderíamos dizer que a natureza problemática social de alguns fatos e/ou situações não é em si uma característica do mesmo fato/situação, mas sim o resultado da atribuição de significado, e das regras e sanções decorrentes; a "história da loucura da época clássica" (Foncault, 1976) é exemplar a este respeito.

Os problemas sociais, que agora podemos definir como o que aparece indesejável e/ou injusto e/ou perigoso e/ou ameaçador de algumas instituições (no sentido mais amplo do termo) que compõem a sociedade e que então devem requerer, em termos de controlo social e através dos vários órgãos responsáveis, uma atenção particular e formas de intervenção. São também definíveis para além do que se afirmou precedentemente, que reenvia mesmo não querendo para as lógicas do desvio (Matza, 1976, sp.), de maneira quase exclusiva e excludente como "o que, pelo menos tal como aparece, anda a perturbar de fato (objetivamente e/ou como percepção) uma ordem não só social mas também cultural, percebida como tendencialmente morfostática e que poderia porém causar danos de sistema de uma certa complexidade".

Uma mistura, portanto, nem sempre fácil de interpretar de condições objetivas e subjetivas, que Merton (1966) já tinha identificado distinguindo entre a dimensão quantitativa da apreciação social dos problemas e aquela qualitativa (coletividade como grupo social funcionalmente significativo), quer dizer alguma coisa que tenha um interesse substancialmente geral, mas de qualquer forma testemunhada por grupos qualificados/qualificantes, os quais afetam (como modalidades ora fortes, ora menos incisivas, ora oscilantes) os comportamentos generalizados da sociedade; esses grupos, 
como veremos mais adiante, concernem diretamente o conceito mais contemporâneo de arena.

Saffirio (1967, p. 270) salienta como podem subsistir (mesmo contemporaneamente) problemas sociais que imediatamente, enquanto existentes como "um consenso completo sobre certos valores sociais e relativos modelos e normas de comportamento", se evidenciam e assim compreendidos (e é por isso que as arenas têm principalmente a função de avaliação e não de identificação ou construção) e outros que não aparecem imediatamente como tal, mas que se tornam mesmo (e se) na medida em que as próprias arenas os definem, os salientam ao interesse mais geral.

Será oportuno, neste ponto, redefinir uma hipótese classificatória dos problemas sociais: tendo em conta as teorias de Sillis (Saffirio, 1967) podemos distinguir os referidos problemas em:

a) Problemas de "patologia social", como alcoolismo, droga, pedofilia, formas organizadas de violência;

b) Problemas de desorganização social, como epidemias, pobreza, marginalização, analfabetismo;

c) Problemas de disfunção social, como corrupção, criminalidade, prostituição, racismo, etc.

Esta hipótese de classificação (como de fato a maioria das classificações) é na realidade muito relativa no sentido em que, conforme o critério base classificatório assumido, os problemas sociais poderiam ser alocados de maneiras diferentes.

Uma última consideração sempre acerca da lógica classificatória: na medida em que se multiplicaram as reflexões acerca da complexidade social e de algumas fenomenologias variadas, mas todas relevantes, que se ligam de fato e/ou de direito à área dos problemas sociais como a violência nos estádios,o uso do álcool por menores,o desaparecimento de crianças,o analfabetismo/alfabetização de camadas 
da população,(e, embora pareça estranho, pelo menos em relação com as outras questões) fumar em público. Estas situações, tendo em conta prioritariamente as causas prováveis, tornam-se, às vezes, dificilmente catalogáveis de maneira incontrovertível nas tipologias acima descritas (e mesmo supondo que "se deva" catalogar sempre).

\section{A produção social da realidade}

Como acima mencionado, os problemas tornam-se sociais quando são (re)conhecidos como tal; por outras palavras portanto, é só no momento em que se substanciam como verdadeiros e reais objetos culturais que existem, embora, pelo menos de acordo com uma abordagem puramente quantitativa, já desde o seu nascimento se refiram a uma realidade não indiferente.

Vamos examinar em seguida alguns percursos de produção cultural dos problemas sociais; em primeira instância, é possível traçar uma hipótese geral que pode ser descrita como um processo feito de imagem-necessidades/expectativas-instituições:

a) A imagem, uma das componentes-constituintes elementares da sociedade, é aquele quid pelo qual o indivíduo conhece a realidade e a comunica a "alter", representando esta a experiência quotidiana da própria realidade.

b) É muito provável que a compreensão da ação social se realize mesmo através da análise da expectativa, fenômeno muitas vezes objetivo para o sujeito, mas muito subjetivo" na realidade.

Como acima se mencionou (Mongardini, 1993; 1995), o comportamento social é governado pela tensão que se constrói em relação a eventos esperados com mais probabilidade do que outros, ou cuja realização já foi repetida várias vezes. Mas como se constitui uma expectativa (ou um sistema de "expectativa")? 
A construção acontece através de uma espécie de "microprocessos" compostos por:

- uma série de pré-condições (informações, imagens, etc.) de base;

- o projeto (ou prefiguração) que o ator social configura;

- a interação estratégica, constituída, na prática, por subexpectativas.

Como características estruturais, a expectativa fornece ao ator social a tensão para agir para um determinado fim.

Finalmente devemos considerar como a mesma expectativa pode ser (ou tornar-se) causa de efeitos não desejados, ou de efeitos atípicos da ação social como, por exemplo, "a profecia que se cumpre por si própria".

Que as expectativas (ou melhor, o horizonte de expectativas) são um fator constitutivo fundamental para a construção da realidade e para a ação social, foi também reafirmado por Griswold (1997) que, desenvolvendo em termos sociológicos anteriores formulações neste sentido da teoria estética, confere uma grande importância ao horizonte de expectativas na atribuição de significado aos acontecimentos e às situações, "de particular interesse para o sociólogo é a virtude adicional que este modelo tem de oferecer ricas possibilidades de comparação". Consideremos o que à primeira vista parece ser não um objeto cultural, mas um evento fatual dramático: a morte de uma criança. Nos Estados Unidos um evento desse tipo é considerado absolutamente trágico, um acidente horrível, uma intrusão de caos na previsibilidade das nossas vidas. A insignificância de uma morte deste gênero pode tornar-se significativa e tornar-se num objeto cultural - pondo-a no nosso horizonte de expectativas em relação às crianças: estas têm um valor individual, são amadas e raramente morrem. A morte de uma criança é, portanto, uma anomalia horrível que anula cada expectativa consolidada.

Em uma favela brasileira, por outro lado, a morte de uma criança tem um significado completamente diferente, os pais que vivem nos miseráveis aglomerados urbanos na 
periferia de uma cidade do nordeste do Brasil colocam a morte de uma criança em um horizonte de expectativas definido pela extrema pobreza, violência, impotência e pela normalidade do evento da morte das crianças antes que elas comecem a viver. Considerando este horizonte, essas mães (e às vezes também os pais, embora os homens sejam geralmente ausentes) reagem à morte de uma criança com fatalismo e uma quase total ausência de emoção. Esses pais brasileiros consideram os filhos e os rapazes como seres humanos potenciais não reais. Para indivíduos com um horizonte deste género, a morte de uma criança não significa "um dos nossos filhos morreu", mas uma criatura não destinada a viver - que não tinha a habilidade de viver, dizem os brasileiros - foi-se embora: “Era um anjo, não um ser humano, e voltou para o céu".

A atenção prestada às diferentes interpretações que as pessoas constroem de um mesmo objeto cultural pode relevar a presença de questões sociais fortemente radicais (Mongardini, 1993; 1995).

Além das expectativas, as necessidades:

[...] a teoria das necessidades é uma das possíveis interpretações da sociedade (e, do nosso ponto de vista, do subsistema social de organização). Deste ponto de vista a sociedade apresenta-se como uma estrutura de resposta às necessidades (articulada, portanto em instituições) posta em prática por indivíduos que, mesmo em função destes, adotam um comportamento específico e repetido em um espaço estruturado real, [...] a necessidade manifesta-se como uma sensação de falta que é simultaneamente estímulo para a pesquisa de meios 
considerados idôneos ou úteis para a própria satisfação. Mas a necessidade não é elemento patológico da vida; tem pelo contrário uma natureza fisiológica: as necessidades são coextensivas à vida e viver significa sentir necessidades. Todo o comportamento está portanto ligado (mas não no sentido determinístico) pela pesquisa de meios para a satisfação das necessidades e a vida aparece como um intercâmbio entre um mundo interno ao indivíduo em que se multiplicam os estímulos e um mundo externo que representa a constrição e a restrição."

(Mongardini, 1995, p. 491).

Finalmente, as instituições, particularmente no que diz respeito aos meios de comunicação de massa, se é verdade que "o que nós sabemos da nossa sociedade e em geral do mundo em que vivemos, sabemo-lo através dos mass media" (Luhmann, 2000, p. 15).

Sucessivamente será amplamente analisado o papel dos meios na construção da realidade; aqui será suficiente citar algumas “áreas" de produção medial;

- os mídia incidem de fato nos conteúdos do esquema de representação da realidade principalmente através da difusividade autoproduzida numa cadeia, como já dito, de referências e filmagens (longitudinais e latitudinais) sem fim: o exemplo do "estábulo mais fotografado da América" é certamente indicativa (Livolsi, 2000);

- os mídia incidem nas formas de conhecimento, ou seja contribuem fortemente para estruturar o nosso saber através de imagens produzidas/reproduzidas (sempre segundo os códigos, as linguagens dos mesmos mídia) e "formatadas" de maneira certamente não neutral, nem pouco relevante 
relativamente ao decoding, mas transferindo-se em profundidade nas nossas categorias interpretativas, nas nossas representações sociais (Moscovici-Farr, 1989);

- finalmente, os mídias podem da mesma maneira fortemente determinar espaços de significação, construindo (pelo menos em grande parte) muitas vezes o lugar onde "as mentes habitam" (Livolsi, 2000, p. 34) e pelo que é impossível não serem influenciadas.

Nestas circunstâncias, quais são as lógicas, os mecanismos, os atores da produção sociocultural da realidade? Pode-se supor um modelo explicativo (mesmo "de grelha larga") de como hoje, numa situação caracterizada por fenomenologias incertas (Bauman, 1998), alguns objetos culturais façam carreira e, de funcionais ou simplesmente situacionais, se tornem culturais nas próprias esferas de referência ou, até, numa geral concepção do mundo.

Consenso especial parece receber o chamado "modelo de Hirsch" (Griswold, 1997, pp. 102-108; Sciolla, s/a, pp. 216-217).

Este modelo constitui uma hipótese cada vez mais interessante e rico de conteúdos e implicações (explícitas, mas também implícitas) acerca de como hoje funciona o mecanismo de produção da cultura, da socialidade consideradas como dois aspectos de uma única realidade, um conjunto sistêmico indiferenciado, mas diferenciável por uma espécie de "artifício metodológico" (Archer, 1997), que se produz coletivamente (e reproduz-se) através da interação que depois, através da linguagem que "liga diferentes zonas no interior da realidade da vida quotidiana, as integra num todo significativo" (Berger-Luckmann, 1969, p. 63). Hirsch constrói um processo composto por quatro elementos constitutivos (os criadores, as organizações, os mídia, o público), três estruturas de filtragem (comparável ao gatekeeping medial) e um duplo feedback; em síntese, o modelo pode ser descrito no seguinte modo: 
a) A hipótese inicial baseia-se no fato que, numa sociedade altamente diferenciada e complexa, se pode de qualquer maneira encontrar por um lado uma incerteza generalizada na procura social de produção de si através do que Griswold define objeto cultural, ou seja um significado partilhado incorporado numa forma audível, visível ou tangível, ou que pode ser articulada" (Berger-Luckmann, 1969, p. 26). A esta vasta procura corresponde igualmente (por um continuum causa/efeito) uma vasta oferta, quer dizer uma abundância (talvez mesmo um excesso) de produção sociocultural;

b) Nem tudo o que é produto chega ao seu destino (ou seja volta para a sociedade no seu conjunto), visto que a produção é filtrada (escolhida, seleccionada) por outros fatores processuais como as organizações de referência da área produtiva (organizações políticas, religiosas, artísticas, etc.) e os mídia; ambos os fatores seleccionam o material não só através de lógicas e processos específicos, mas sobretudo através do critério do código binário (Luhmann, 2000) típico de cada sistema que inclui/exclui a realidade de acordo com as suas necessidades autorreferenciais;

c) O que chega ao público também é filtrado, escolhido às vezes através de critérios mais psicológicos como a "seletividade" ou a "dissonância cognitiva" (Cheli, 1996), às vezes mais sociológicos como estilos de vida, modas. Portanto o público, mesmo através da fruição/não fruição dos objetos, responde em termos de feedback comunicativo, quer dizer indicando as próprias escolhas/preferências e, por conseguinte, orientando (mas não de maneira rigidamente causal) a produção/difusão dos outros sistemas.

O modelo de Hirsch como lembra Griswold, nasceu para os "produtos culturais de massa tangíveis", mas pode ser aplicado, com as necessárias/oportunas modificações, a qualquer produção sociocultural: 
[...] se, por exemplo, tivéssemos que assumir uma certa posição teológica como objeto cultural (por exemplo uma leitura feminista da Bíblia), poderíamos pensar numa confissão religiosa em termos parecidos a um sistema de indústria cultural, percebendo as mensagens teológicas como produtos ou, por outras palavras, como objetos culturais. O subsistema técnico consistiria assim em licenciados em teologia à procura de uma posição. O subsistema gerencial dependeria das igrejas da confissão (por simplicidade, podemos assumir uma comunidade congregacionalista como os batistas, onde cada igreja escolhe ou chama os seus pastores). Os recém-diplomados pelo seminário dirigem-se neste caso aos conselheiros espirituais do seminário, pedindo-lhes para os ajudarem a encontrar uma colocação, enquanto as igrejas têm as próprias interfaces nas comissões de pesquisa pastoral que avaliam o talento. O feminismo de um aspirante a pastor que pode ser um recurso ou uma honra para uma certa igreja, como o estilo executivo de um artista pode atrair ou não o interesse dos talent scouts de uma loja de discos. Uma vez que pastor começou o seu ministério - ou seja foi assumido por uma organização de produção cultural no subsistema gerencial - a sua mensagem, através dos sermões, dos rituais e do exemplo pastoral, chegará aos consumidores, os membros da congregação. Um sistema institucional como a imprensa local pode pôr em evidência o pastor e as suas inovações; em alguns casos esporádicos, as igrejas dirigem-se a um mais sofisticado uso da rádio ou da televisão para transmitirem sermões e serviços. O meio mais importante, porém,é a comunicação face a face. O feedback chega ao pastor e aos dirigentes da igreja diretamente da congregação. Um dos feedbacks mais importantes, semelhante à venda de bilhetes, vem das mudanças nos níveis de participação. Se o pastor for popular, se a sua combinação inovadora 
entre ortodoxia bíblica e feminismo funcionar bem, a palavra espalha-se na comunidade e a congregação cresce. Caso contrário, se estiver de facas arrancadas com a sua congregação, se a sua teologia e a religiosidade dos membros da congregação não se adaptarem mutuamente, estes podem votar, não indo à igreja, fazendo diminuir assim o nível de participação e empurrando a igreja para retornar ao subsistema técnico em busca de novos talentos."

(Griswold, 1997, pp. 107-108)

\section{Uma hipótese de trabalho}

Tentemos agora construir uma hipótese de trabalho relativamente a como os problemas sociais se tornam efetivamente tais ou talvez não muito, partindo como pressuposto da cultura, dimensão constitutiva da nossa experiência de vida, que impõe significados a um universo que senão seria caótico e casual" (Griswold, 1997, p. 133). Os sistemas culturais transformam eventos e coisas em objetos culturais com específicos significados para cada cultura e no âmbito de cada cultura, e isso explica como alguns fenômenos do mundo social são considerados importantes, transformados em objetos culturais e finalmente em problemas sociais, enquanto outros permanecem no esquecimento. Mas se a cultura pode atrair a atenção acerca dos problemas sociais, pode ela também às vezes gerar o problema?

Comecemos, antes de mais nada, considerando como os fatos que acontecem tornam-se em objetos culturais: a criação de um objeto cultural, é semelhante à criação de um fato, definível como a relação, criada pela interpretação, entre um fato e uma estrutura. Mas como podem os fatos tornar-se objetos culturais identificados como problemas sociais? 
Os problemas sociais, como já dissemos, são os produtos de um processo de definição coletiva. Este ponto de vista recusa a lógica segundo a qual os problemas sociais são objetivos e identificáveis automaticamente. Afirma que eles são produtos de sentimentos coletivos em vez de espelhos de condição objetiva da sociedade: de fato, há muitas situações na sociedade que poderiam ser percebidas como problemas sociais, mas não são definidas como tal, uma teoria que os considera como meros reflexos de condições objetivas não pode explicar porque algumas condições são definidas como problemas, controlando a maior parte da atenção da sociedade enquanto outras, igualmente significativas, não são assim.

Por que, por exemplo, a condição das populações autóctones do Brasil (que sofrem pela contínua destruição das culturas, do ambiente e das próprias populações) recebe menor atenção do que a condição dos animais usados nos laboratórios para a investigação científica? Por que há condições e eventos no "Terceiro Mundo" que alteram a vida de milhões de pessoas e que, aliás, no mundo ocidental se tornam objeto de uma única e precipitada atenção sobretudo superficial por parte do público só durante as crises políticas?

E o escasso (ou muito flutuante) interesse pela morte de florestas, como se o ambiente no seu conjunto fosse um problema de outros?

A extensão dos danos nestes casos não pode, por si só, explicar essas diferenças, e não é suficiente dizer que a mesma destas situações se torne num problema social porque é "muito importante". Todos estes problemas são importantes ou no final podem ser vistos como tal. Por fim, é inútil reivindicar simplesmente esses mesmos problemas como muito mais comerciáveis do que outros.

Muitos autores, como Schneider e Blumer (apud Hilgartner Bosk, 1988) propuseram uma interessante analogia com os modelos de história natural para explicar as etapas da carreira dos problemas sociais. Utilizando assim a moldura da história natural, estas 
pesquisas desenvolveram estudos de caso que traçam a progressão do problema social através de uma sequência de fases: início, fusão, institucionalização, fragmentação e fim. Mas, mesmo concordando que esses modelos são considerados para ser, de qualquer maneira, estimulantes, a ideia de uma sucessão ordenada de etapas é limitativa: muitos problemas existem simultaneamente em diferentes etapas de desenvolvimento, e os modelos de progressão de uma etapa para outra variam suficientemente para ter a pretensão que existe uma carreira típica; depois as interacções entre problemas estão centradas pelo processo de definição colectiva (apud Hilgartner-Bosk, 1988, p. 54).

Esta análise centrou de fato a atenção nos problemas individuais, e esta ênfase contribuiu para a subestimação das duas características críticas do mesmo processo, quer dizer que existem problemas sociais em relação a outros e que eles estão imersos numa complexa institucionalização de formulação do problema.

Hilgartner e Bosk propõem um método diferente e identificam vias para o estudo sistemático dos fatores e das forças que dirigem a atenção do público para o mesmo e longe de outras condições objetivas; os nossos autores definem um problema social como uma condição putativa ou situação que é etiquetada como problema nas arenas do discurso público e da ação (1988, pp. 53-78), e isso através de um amplo âmbito de literatura sociológica geral e de literatura específica acerca do processo interpretativo nos mass media; utilizam portanto a teoria dos network organizativos, submetendo a influência e as inter-relações entre as instituições e os network sociais, às definições de problema enquadradas e apresentadas publicamente. Relacionam-se depois em particular à agenda setting, apontando que o focus original (o processo que organiza a agenda para as decisões nos fóruns oficiais) consiste em limitar as escolhas dos outros (o processo que estrutura o que diz respeito ao coletivo nas arenas públicas); finalmente, ligam este modelo a uma estrutura ecológica, não para proporem relações determinísticas, mas para atingirem a totalidade do recurso que os atores sociais 
utilizam na construção das definições do problema. Tudo isso fornece a base para a profunda complexidade do processo de definição coletiva, que envolve os processos sócio-psicológicos, organizacionais, políticos e culturais com uma influência penetrante na ação social a vários níveis.

Este modelo complexo contém alguns elementos constitutivos como:

a) O processo dinâmico de competição entre os membros de uma ampla população que reivindica os problemas sociais;

b) As arenas institucionais que servem como condicionamentos ambientais, onde os problemas sociais competem uns com os outros em termos de atenção e de desenvolvimento.

Os problemas potenciais não só são regidos pela sua natureza objetiva, mas também por um processo altamente seletivo em que competem para receber atenção pública por parte da sociedade: uma fração de problemas potenciais é apresentada publicamente por grupos ou indivíduos que os definem como problemas; estes sujeitos, provenientes de muitos setores da sociedade, podem ter objetivos muito diferentes: grupos de interesse, políticos e movimentos sociais organizados podem tentar promover as mudanças sociais ou reformas mesmo através do uso dos problemas sociais;

c) As capacidades arrastadoras destas arenas, como limite do número de problemas que podem ganhar ao mesmo tempo a atenção generalizada;

d) Os princípios de seleção, ou os fatores institucionais, políticos e culturais que influenciam a probabilidade de sobrevivência das formulações competitivas do problema;

e) Os modelos de interação entre arenas diferentes;

f) Os networks das operabilidades que promovem e tentam controlar alguns problemas especiais e cujos canais de comunicação formam as diferentes arenas. 
Como primeiro passo para compreender a natureza do processo de definição coletiva, é necessário notar que existe uma grande massa de potenciais problemas, ou seja, situações e condições putativas que poderiam ser concebidas como tal e que esta massa é altamente estratificada. Uma fração muito pequena cresce no interior dos problemas sociais atingindo a celebridade, ou seja, os temas dominantes de dissertação política e social, enquanto um amplo número equivalente desenvolve no interior outras problemáticas, limitadas comunidades de profissionais, ativistas e interessantes grupos de trabalho mantêm vivos estes problemas à margem do debate público.

Dentro de cada área problemática maneiras diferentes de enquadrar a situação podem competir, para serem aceites como a versão autorizada da realidade, portanto uma grande recolha de problemas (da gravidez na adolescência à escassez de doação de órgãos) compete para a atenção pública, através de um complexo processo de seleção que define as prioridades acerca do que deveria ser considerado como importante.

A definição coletiva dos problemas sociais não acontece em abstrato na sociedade ou na opinião pública, mas, como já foi dito nas arenas públicas e particulares em que os problemas sociais são enquadrados e se desenvolvem como tal; estas arenas incluem os ramos executivos e legislativos do governo, os mídia (new e old), as campanhas políticas, as ações dos movimentos coletivos, as organizações religiosas, as sociedades profissionais e/ou fundações privadas: é de fato nestas situações que os problemas sociais são discutidos, selecionados, definidos, enquadrados, dramatizados: confeccionados e apresentados ao grande público. Embora existam diferenças substanciais entre estas arenas, uma característica fundamental é que cada arena tem uma capacidade de carga que limita o número de problemas sociais que pode entreter em cada vez; resulta claro também que o número de situações que poderiam ser interpretadas potencialmente como problemas é tão grande que pode ser virtualmente infinito, enquanto o espaço e o tempo para apresentar os problemas publicamente é 
completamente limitado, e é esta discrepância, entre o número de problemas potenciais e as dimensões do espaço público que os podem receber, que torna a concorrência entre os problemas tão crucial e central no processo de definição colectiva.

\section{REFERÊNCIAS}

ARCHER, Margaret. La morfogenesi della società. Milano: Franco Angeli, 1997.

BAUMAN, Zygmunt. La società dell'incertezza. Bologna: Il Mulino, 1999.

. Modernità liquida. Roma-Bari: Laterza, 2002.

BEATO, Fulvio. Le teorie sociologiche del rischio. In: DE NORDIS, Paolo (Org.). Le nuove frontiere della sociologia, Roma: Carocci, 1998.

BECK, Ulrich. La società del rischio. Roma: Carocci, 2000.

BERGER, Peter; LUCKMANN, Thomas. La realtà come costruzione sociale. Bologna:Il Mulino, 1969.

BERGER, Peter; BERGER, Brigitte. Sociologia. Bologna: Il Mulino, 1995.

BERGER, Brigitte; KELLNER, Hansfried. The homeless mind. London: Penguin, 1973.

BOVONE, Laura. In tema di postmoderno. Milano: Vita e Pensiero, 1990.

. Vivere in società. Napoli: Liguori, 1996.

CAILLOIS, Roger. I giochi e gli uomini. Milano: Bompiani, 1981.

CENSIS (org). Dossier "Illecito". Quindicennale di note o commenti, 4, 1985.

CESAREO, Vincenzo. Sociologia. Concetti e tematiche. Milano: Vita e Pensieri, 1988.

COLOMBO, Enzo. Rappresentazioni dell'Altro. Milano: Guerini e Associati, 1999.

CRESPI, Franco. Le vie della sociologia. Bologna: Il Mulino, 1985.

. Manuale di sociologia della cultura. Roma-Bari: Laterza, 1996.

DE VITA, Roberto. Incertezza e identità. Milano: Franco Angeli, 1999.

. Identità e dialogo. Milano: Franco Angeli, 2003.

DAL LAGO, Alessandro. I nostri riti quotidiani. Genova: Costa e Nolan, 1995.

DI CRISTOFARO LONGO, Gioia. Identità e cultura. Roma: Studium, 1996.

ECO, Umberto. Apocalittici e integrati. Milano: Bompiani, 1964. 
ECO, Umberto. Trattato di semiotica generale. Milano: Bompiani, 1975.

GALLINO, Luciano. Dizionario di sociologia. Torino: Utet, 1993.

GEERTZ, Clifford. Interpretazioni di cultura. Bologna: Il Mulino, 1987. . Anti-antirrelativismo. Roma: Il Mondo 3, 1995.

GRISWOLD, Wendy. Sociologia della cultura. Bologna: Il Mulino, 1997.

HILGARTNER, Stephen; BOSK, Charles. The rise and fall of social problems. In: American Journal of Sociology, n. 9, 1988.

JEDLOWSKI, Paolo. Storie comuni. Milano: Bruno Mondatori, 2000.

KARDINER, Abram. L'individuo e la sua società. Milano: Bompiani, 1965.

KLUCKHOHN, Clyde; KROEBER; Alfred. Il concetto di cultura. Bologna: Il Mulino, 1972.

LEMERT, Edwin. Social problems, In: SILLS, David. (ed.). International Encyclopedia of Social Sciences, McMillan: New York, 1968.

LUHMANN, Niklas. Sistemi sociali. Bologna: Il Mulino, 1990.

. La realtà dei media. Milano: Franco Angeli, 2000.

LYOTARD, Jean François. Il postmoderno spiegato ai bambini. Milano: Feltrinelli, 1987.

MARINELLI, Alberto. La costruzione del rischio. Milano: Franco Angeli, 1993.

MARTELLI, Stefano. Sociologia dei processi culturali. Brescia: La Scuola, 1999.

MATZA, David. Come si diventa devianti. Bologna: Il Mulino, 1976.

MELUCCI, Alberto (Org.). Parole chiave. Roma: Carocci, 2000.

MONGARDINI, Carlo. La conoscenza sociologica. Genova: Ecig, 1995.

. La cultura del presente. Milano: Franco Angeli, 1993.

MOSCOVICI, Serge; FARR, Robert (Ed.). Rappresentazioni sociali. Bologna: Il Mulino, 1989.

PAWLUCH, Dorothy. Social problems. In: KUPER Adam; KUPER, Jessica (Ed.). The social science Encyclopedia. London: Routledge, 1996.

SAFFIRIO, Luciano. La definizione del problema sociale. In: Quaderni di sociolog, 1967.

SCATIZZA, A. L'interazione sociale translocale. In: Comunicando, n. 3, 2003.

SCIOLLA, Loredana. Sociologia dei processi culturali. Bologna: Il Mulino, 2002. 
SCIOLLA, Loredana. Il concetto di identità in sociologia. In: RASS. Ital. di Sociologia, n. 3, 1985.

SEYMOUR-SMITH, Charlotte. Dizionario di antropologia. Firenze: Sansoni, 1991.

SMELSER, Neil. Il comportamento collettivo. Firenze: Vallecchi, 1968.

TAGLIOLI, Ricardo. Problemi sociali, riproduzione, struttura. Milano: Franco Angeli, 1990.

TENTORI, Tullio. Il pregiudizio sociale. Roma: Studium, 1962.

. Il rischio della certezza. Roma: Studium, 1989.

TURNER, Victor. Antropologia della performance. Bologna: Il Mulino, 1993.

UNGARO, Daniele. Capire la società contemporânea. Roma: Caro, 2001. 